Artigo de Revisão

\title{
Assistência à saúde da pessoa com deficiência nos serviços públicos de saúde: um estudo bibliográfico
}

Health care of persons with disabilities in public health services: a literature study

\author{
Sheila Cristina Vargas ${ }^{1}$, Robson Resende Dutra ${ }^{2}$, Luis Filipe Haesbaert Barcelos ${ }^{3}$, Lia \\ Gonçalves Possuelo², Suzane Beatriz Frantz Krug². \\ ${ }^{1}$ Prefeitura Municipal de Cruz Alta, Cruz Alta, RS, Brasil. \\ ${ }^{2}$ Universidade de Santa Cruz do Sul, Santa Cruz do Sul, RS, Brasil. \\ ${ }^{3}$ Hospital São Vicente de Paulo Cruz Alta, Cruz Alta, RS, Brasil.
}

Submissão: 29/08/2016

Aceite: 03/10/2016

\section{RESUMO}

shemestrado@hotmail.com

Justificativa e objetivos: A assistência à saúde às pessoas com deficiência (PcD) deve ser garantida pelo Estado, profissionais de saúde e comunidade envolvida, abrangendo uma visão multidisciplinar. Este estudo tem como objetivo discutir sobre a assistência às pessoas com deficiência nos serviços públicos de saúde. Métodos: Trata-se de uma revisão bibliográfica do tipo estudo descritivo com publicações científicas nos sites de busca Scielo, LILACS e Pubmed a partir dos descritores: acessibilidade, pessoas com deficiência, acesso aos serviços de saúde, totalizando 514 artigos,onde se enquadram 22 nas categorias temáticas abordadas. Resultados: A acessibilidade é resultado da disponibilidade de profissionais e serviços de saúde, assim como, o acesso das Pessoas com Deficiência (PcD) a esses serviços ofertados. É necessário o planejamento de ações por parte da equipe multidisciplinar, para que se busque a minimização das desigualdades frente a barreiras comportamentais, arquitetônicas, geográficas, o que forma lacunas que impedem uma assistência igualitária, unânime e universal como é preconizado pelo sistema de saúde. Na saúde bucal o princípio de integralidade contempla a ações de promoção, recuperação e reabilitação oral. Conclusão: As ações de promoção da saúde precisam ser incentivadas para que se promova o bem estar do usuário do serviço de saúde e que tais ações ocorram de forma integrada, somando recursos por parte do trabalho integral e multidisciplinar. A acessibilidade aos serviços de saúde em conjunto com ações que visem promover a saúde dos PcDs podem proporcionar maior qualidade na assistência à saúde e maior qualidade de vida.

DESCRITORES: Acessibilidade. Pessoas com deficiência. Acesso aos serviços de saúde.

\section{ABSTRACT}

Background and Objectives: Health care for people with disabilities (PwD) must be guaranteed by the state, health professionals and community involved, covering a multidisciplinary approach. This study aims to discuss the assistance to persons with disabilities in public health services. Method: This is a literature review of the descriptive study type with scientific publications on search sites Scielo, LILACS and Pubmed from descriptors: accessibility, people with disabilities, access to health services, totaling 514 articles, which fall under 22 the themes addressed. Results: Accessibility is a result of the availability of professionals and health services as well as access of Persons with Disabilities these services offered. We need planning actions by the multidisciplinary team, in order to 
seek to minimize the front inequalities behavioral barriers, architectural, geographical, which form gaps that prevent an egalitarian, unanimous and universal care as recommended by the health system. In oral health the principle of comprehensiveness includes the promotion, recovery and oral rehabilitation. Conclusion: Health promotion activities need to be encouraged so that it promotes the welfare of the health service user and that such actions occur in an integrated manner, adding resources from the comprehensive and multidisciplinary work. Accessibility to health services in conjunction with actions aimed at promoting the health of PwD can provide higher quality in health care and higher quality of life.

KEYWORDS: Accessibility. People with disabilities. Access to health services.

\section{INTRODUÇÃO}

Estima-se que 23,9\% da população brasileira vivem com algum tipo de deficiência que, conforme a Pesquisa Nacional de Saúde (PNS) pode ser classificada em quatro tipos: deficiência intelectual, auditiva, física e visual. A PNS aponta a deficiência visual como a mais comum entre os brasileiros, atingindo cerca de 3,6\% da população, com seu maior número de casos concentrados na região sul do Brasil. ${ }^{1}$ O Decreto 3.298 de 20 de dezembro de 1999 descreve a deficiência como a perda ou anormalidade de uma estrutura ou função psicológica, fisiológica ou anatômica que pode gerar incapacidade ou dificuldade para o desempenho de uma atividade, dentro do padrão considerado normal para o ser humano. ${ }^{2}$

Segundo estatísticas há baixa procura por serviços da rede pública de saúde por pessoas portadoras de alguma deficiência. De acordo com a PNS os índices da procura por este tipo de serviço se configuram em menos de $5 \%$ por parte dos deficientes visuais; $18,4 \%$ dos deficientes físicos, e 30\% dos portadores de deficiência intelectual. Outro dado importante é que os percentuais mais elevados de deficiência intelectual, física e visual foram encontrados, na população que possui somente o ensino fundamental ou sem instrução nenhuma. Estima-se que esse grupo está submetido a várias barreiras, dentre elas a acessibilidade aos serviços públicos de saúde, dificultando assim sua integração social ${ }^{1}$. No Brasil, 15.687 estabelecimentos de saúde informaram ter rampa de acesso ao seu interior, o que favorece a acessibilidade. Na região sul são 3.294 estabelecimentos com acesso facilitados sendo 1.319 no estado do Paraná, 841 no estado de Santa Catarina e 1.134 no Rio Grande do Sul.

Em conformidade com a Lei Orgânica de Saúde nº 8.080/90, a implementação da Política Nacional de Saúde da Pessoa com Deficiência, instituída pela Portaria MS/GM no 1.060, de 5 de junho de 2002, que firma princípios importantes do Sistema Único de Saúde 
(SUS) como a universalidade, integralidade e equidade, garante aos portadores de algum tipo de deficiência o direito assegurado à saúde. ${ }^{2}$

Mesmo com mais de 20 anos de implementação do SUS, ainda falta conhecimento sobre seus direitos e acesso a rede pública de saúde em relação aos pacientes com deficiência. Essa falta de informação dificulta a construção de indicadores importantes para a avaliação e acompanhamento das necessidades desta população. ${ }^{3}$ A assistência à saúde às pessoas com deficiência deve ser garantida pelo Estado, profissionais de saúde e comunidade envolvida, abrangendo uma visão multidisciplinar. Uma equipe multidisciplinar, com visão justa e igualitária quanto ao direito à saúde, tende a apresentar resultados mais efetivos na assistência e conseqüentemente para a qualidade de vida destes pacientes. ${ }^{2}$ Diante deste contexto, o presente estudo de revisão bibliográfica como objetivo discutir sobre como se figura a assistência às pessoas com deficiência nos serviços públicos de saúde.

\section{MÉTODOS}

O artigo abrange um estudo de revisão bibliográfica realizada com artigos publicados entre os anos de 2013 a 2016, em periódicos nacionais e internacionais disponíveis nas bases de dados SCIELO, LILACS e PUBMED. Para esta busca, os descritores utilizados foram acessibilidade, pessoas com deficiência, acesso aos serviços de saúde, sendo localizados no portal da Biblioteca Virtual em Saúde.

Após a busca nas bases de dados, procedeu-se a leitura dos resumos dos artigos para selecionar os textos que atendiam aos critérios de inclusão de acordo com as áreas temáticas abordadas e publicados nos idiomas de inglês ou português. Foram eliminados os artigos duplicados, os que não estavam de acordo com o tema proposto e os que não se apresentavam na integra nos sites da pesquisa.

Após essa etapa, os dados foram aglutinados e discutidos à luz de referenciais teóricos, nas seguintes categorias temáticas: acessibilidade aos serviços públicos de saúde; qualidade da assistência de saúde aos PcDs e acesso à saúde bucal.

\section{RESULTADOS E DISCUSSÃO}

Trata-se de um método de revisão integrativa. Segundo Mendes (2008) "A revisão integrativa inclui a análise de pesquisas relevantes que dão suporte para a tomada de decisão e 
a melhoria da prática clínica, possibilitando a síntese do estado do conhecimento de um determinado assunto, além de apontar lacunas do conhecimento que precisam ser preenchidas com a realização de novos estudos". ${ }^{4}$ A revisão seguiu critérios específicos como a seleção dos artigos, definição da pergunta de revisão (Quais são as ações que o serviço público de saúde realiza para melhorar a qualidade da assistência de saúde e a acessibilidade a esses serviços aos PcD!), análise crítica dos estudos, discussão dos resultados e a interpretação de dados. ${ }^{5}$ A Figura 1 apresenta uma síntese dos dados encontrados na base de dados através da Biblioteca Virtual em Saúde e na Biblioteca eletrônica Scielo.

Figura 1 - Fluxograma da seleção de artigos da revisão bibliográfica.
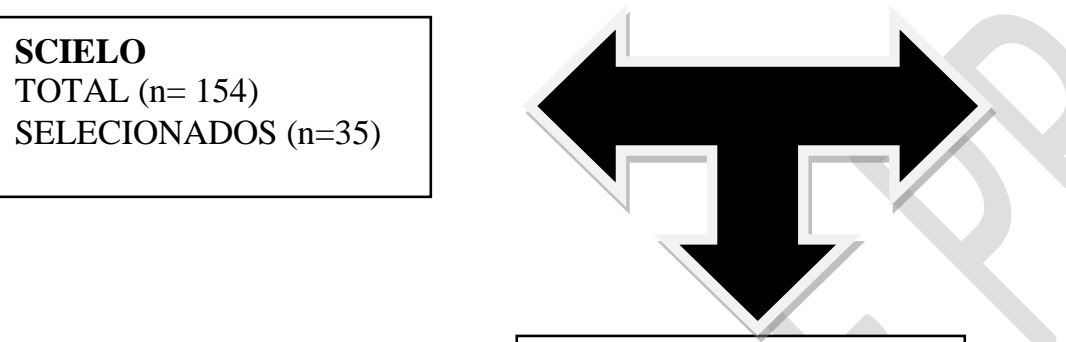

LILACS

TOTAL $(\mathrm{n}=2.165)$

SELECIONADOS $(\mathrm{n}=427)$

\section{PUBMED}

TOTAL $(\mathrm{n}=203)$

SELECIONADOS $(\mathrm{n}=52)$

Fonte: Medline, Lilacs e Scielo 2013-2016.

Foram selecionados 514 artigos nas bases de dados, onde 84 encontrados eram duplicados e 240 se enquadravam dentro dos descritores propostos. Destes, 22 cumpriam os critérios de inclusão.

\section{Qualidade da assistência de saúde aos PcD}

A exclusão das pessoas com deficiência poderia ser em menor número quando se avalia os grupos existentes na sociedade brasileira, facilitando o acesso aos serviços públicos que mais necessitam. ${ }^{6}$ Todavia, existem muitos limites em relação a garantir acesso e atendimento, tendo como referencia o direito à saúde. Segundo a Organização mundial de Saúde (OMS), cerca de $10 \%$ da população em países subdesenvolvidos necessitam de uma atenção especial, que na maioria das vezes tem que ter o cuidado com uma equipe 
multidisciplinar com abordagem interdisciplinar, para poder ter um atendimento mais seguro e eficaz, visando o bem-estar ao paciente. ${ }^{7}$

No entanto, existe uma resistência para o atendimento de pacientes com necessidades especiais por parte dos profissionais da saúde que pode ser atribuída à falta de treinamentos adequados, conhecimento, confiabilidade, sensibilidade, além da inadequação da remuneração. Além disso, muitas vezes são necessários alguns equipamentos especiais para facilitar e agilizar o tratamento proposto e que não estão disponíveis no serviço público. ${ }^{8,9}$

São questões agravadas quando relacionada aos problemas de ansiedade e apreensão por parte dos familiares. Isto acontece por existir números problemas presentes na rotina diária bem como, dificuldade dos pacientes se expressarem, dificuldades de transporte, falta de acesso e principalmente de informações sobre seus direitos. ${ }^{10}$

Houve um grande avanço no Brasil em relação á qualidade de vida dos PcD e o mesmo pode ser atribuído à implementação de diretrizes que possibilitam a promoção e educação em saúde além de aumentar novas oportunidades no mercado de trabalho. ${ }^{11,12}$

No entanto, há muitas barreiras e dificuldades na atenção primária dentre elas a inacessibilidade e a inadequação da estrutura física das Unidades Básicas de Saúde (UBS). Além disso, devemos considerar os problemas com os transportes coletivos sem adaptação, demora no atendimento e ambientes lotados que dificultam acesso ao serviço. ${ }^{13,14}$

Todos esses fatores ocorrem por falta de investimentos nas infraestruturais das unidades básicas e negligência por parte dos gestores em proporcionar um ambiente mais adequado. A falta de acessibilidade tem sido um obstáculo na consolidação do principio básico que é a universalidade aos pacientes com deficiência. ${ }^{15,16}$

\section{Acessibilidade aos serviços públicos de saúde}

A acessibilidade é resultado da disponibilidade de profissionais e serviços de saúde, assim como, o acesso dos PcD a esses serviços ofertados. É necessário planejamento de ações por parte da equipe multidisciplinar, para que se busque minimizar as desigualdades frente às barreiras comportamentais, arquitetônicas e geográficas que formam lacunas que impedem uma assistência igualitária, unânime e universal como é preconizado pelo sistema de saúde. ${ }^{17}$ As ações de promoção da saúde como o atendimento multidisciplinar, visita domiciliares e acompanhamento não só do paciente mais também dos familiares, permitindo um vinculo entre o PcD e a UBS, precisam ser incentivados objetivando o bem estar do usuário do serviço 
de saúde de forma integrada somando recursos por parte de um trabalho integral e multidisciplinar. As dificuldades encontradas são inúmeras, podendo ser citadas a falta de infraestrutura como estacionamento preferencial, rampas de acesso, banheiros adaptados, transporte e sala de espera adequada. Pode-se perceber que essas barreiras aos serviços de saúde limitam o direito das PcD a assistência universal garantida pelo SUS. ${ }^{15-17}$

A Política Nacional de Integração da Pessoa com Deficiência visa à assistência integral e reabilitação, além de fornecer um atendimento que aumente as possibilidades de inclusão. Isto faz com que os gestores tenham um comprometimento com as necessidades de capacitação e qualificação de toda a equipe de saúde. ${ }^{18}$

Sabemos que a qualificação dos profissionais da saúde é de extrema importância, por isso é necessário projetos e programas de atenção permanentes para que viabilizem um melhor preparo facilitando e melhorando as necessidades das pessoas com deficiência. Um ponto importante da equipe de saúde é identificar e cadastrar as pessoas com deficiência, trabalho no qual os agentes comunitários de Saúde podem realizar de forma mais precisa, levando informações importantes para a equipe de saúde. Com isso, as UBS devem criar um ambiente que atenda aos princípios da universalidade, integralidade e equidade provendo também a capacitação dos profissionais da saúde. ${ }^{19-21}$ Com o desenvolvimento dessas praticas de saúde realizada em equipe multiprofissional pode-se contribuir para a construção de ferramentas de promoção da saúde da pessoa com deficiência. ${ }^{21}$

Outro fator importante é a dificuldade dos profissionais em se comunicar com os pacientes, o que pode interferir diretamente na qualidade da assistência, impedindo a formação do vinculo e da confiança entre o profissional e o paciente com deficiência. Conseqüentemente o atendimento será pouco resolutivo, comprometendo a integralidade. ${ }^{22}$ Aprimorar o conhecimento em diversas áreas da saúde irá contribuir para ações com abordagem interdisciplinar, promovendo saúde ao paciente com deficiência. ${ }^{14}$

\section{Acesso à saúde bucal}

Os estudos abordados tem mostrado uma deficiência na saúde bucal dos $\mathrm{PcD}$, grande maioria encontrada na atenção primária, onde aparece como item mais importante a escovação supervisionada, que é realizada na cadeira odontológica. Foram encontradas muitas barreiras que levam o PcD a não procurar o serviço que a atenção primaria oferece. Visto isso, a importância em abordar sobre a saúde bucal tem sido tema relevante na atualidade. ${ }^{23}$ 
Na odontologia, desde 2004, a Política Nacional de Saúde oferece o atendimento a pessoas com deficiência como atenção secundária, por meio de Centro de Especialidades Odontológicas (CEO). Os CEO são estabelecimentos que prestam serviços especializados em saúde bucal, dando ênfase ao diagnostico de câncer de boca, periodontia, cirurgia, endodontia e atendimento ao paciente com deficiência. ${ }^{23,24}$

$\mathrm{Na}$ saúde bucal o princípio de integralidade contempla a ações de promoção, recuperação e reabilitação oral, devolvendo ao paciente com deficiência um sorriso mais bonito e uma melhor qualidade de vida. ${ }^{25}$ Um fator importante para assistência à saúde bucal é a forma como está organizado o sistema, dando ênfase em um sistema mais estruturado e articulado, funcionando através de uma referência e contra referência entre a estratégia de saúde da família e o centro de especialidades. Para que ocorra um bom funcionamento tem que haver comunicação de ambos, garantindo a integralidade em saúde bucal. ${ }^{26}$

Para a odontologia é considerado paciente com necessidades especiais a pessoa que apresenta limitações e dificuldades ou aqueles sem habilidades em realizar higiene bucal correta. Alguns não permitem que outras pessoas como pais, cuidadores realizem a higiene bucal de maneira adequada por apresentarem um comportamento agressivo, ou movimentos involuntários, dificultando a higienização, com isso facilita a não adequação do meio bucal, muitas vezes ocasionando dor, edema, ocasionando a não socialização com outras pessoas da sociedade. $^{27-29}$

Faz parte do atendimento odontológico à pessoa com deficiência não considerar somente o seu corpo patológico, mas sim, um atendimento humanizado e apropriado, facilitando o atendimento, criando vínculo, com atendimento digno para esses pacientes. Inseri-los em um espaço social e familiar, por meio do cuidado mais humanizado, conhecendo a realidade dos mesmos, de forma a proporcionar integralidade na assistência á saúde do paciente com deficiência, contribuindo para o desenvolvimento da saúde bucal, resultando em uma melhor qualidade de vida. ${ }^{29,30}$

\section{CONCLUSÕES}

Toda PcD tem como referência o direito a saúde, fazendo parte disto estão o acesso e a assistência ao atendimento por parte dos profissionais de saúde, que atribuem as dificuldades encontradas por difícil manejo, falta de material adequado e capacitação, proporcionando assim um atendimento resolutivo e promovendo saúde aos PcD. Hoje ainda 
podemos perceber que a acessibilidade não é somente o uso de serviços, mais é conhecimento por parte dos profissionais, equipamentos adequados, transporte adequado e que tenham oportunidades iguais a todo o cidadão.

As inadequações na acessibilidade tem sido o motivo mais importante pelo qual o PcD não procura atendimento aos serviços de saúde. É necessário que as políticas públicas para o PcD sejam colocadas em práticas como ações em saúde e fácil acesso ao serviço, além de facilitar a locomoção para que o PcD tenha um atendimento adequado. Há necessidade de políticas públicas que favoreçam esta população, em uma visão interdisciplinar de assistência à saúde. Na saúde bucal não é diferente, as ações de promoção em saúde tem que visar à busca do PcD e capacitar seus cuidadores para que ocorra uma saúde bucal constante, melhorando sua qualidade de vida.

Assegurar que todo o paciente com deficiência tenha acesso aos serviços públicos de saúde, política especifica e ações de promoção e educação da saúde fazem com que a equidade esteja presente em todos os aspectos da vida em uma sociedade.

O entendimento que se trata de um trabalho a ser realizado a médio e longo prazo pressupõe obter maior visibilidade para a temática no âmbito do sistema de saúde para que a pessoa com deficiência disponha de um serviço que funcione em consonância com os princípios de universalidade e integralidade.

\section{REFERÊNCIAS}

1. IBGE - Instituto Brasileiro de Geografia e Estatística. Censo Demográfico de 2010 [Internet]. [Acesso 20 jun 2016]. Disponível em http://www.ibge.gov.br/home/presidencia/noticias/imprensa/ppts/00000008473104122012315 727483985>

2. BRASIL. Portaria $n^{\circ} 1060$, Lei $8.080 / 90$ de 5 de junho de 2002.Aprova a Política Nacional de Saúde da Pessoa Portadora de Deficiência. Diário Oficial da União 2002; 5 jun.Brasil. Decreto 3.298 de 20 de Dezembro de 1999. Dispõe sobre a Política Nacional para a Integração da Pessoa Portadora de Deficiência, consolida as normas de proteção, e dá outras providências. Diário Oficial da União; 21 dez. 1999.

3. IBGE - Instituto Brasileiro de Geografia e Estatística, PNS- Pesquisa Nacional de Saúde, Ministério da Saúde, Deficiência visual, Deficiência física, Deficiência auditiva, Deficiência intelectual, 2013.

4. Mendes KDS, Silveira RCCP, Galvão CM. Revisão integrativa: método de pesquisa para a incorporação de evidências na saúde e na enfermagem. Texto Contexto Enferm 2008; 17(4):758-64. 
5. Creswell JW. Revisão da literatura. In JW. Creswell, Projeto de pesquisa (M. Lopes, Trad.), Porto Alegre, 2010; 3:48-75

6. Chaves SCL. et al. Fatores associados à integralidade na saúde. Revista de Saúde Pública, 2010; Disponível em: www.scielo.br/rsp

7. Bezerra TV, Silva MA, Maia ER. Acesso da pessoa com deficiência à atenção primária em saúde no Brasil: limites e possibilidades. Caderno de Cultura e Ciência, Ano X, 2015; $14: 2$

8. Cardoso et al. O Acesso ao Cuidado em Saúde Bucal para Crianças com Deficiência Motora. PesqBrasOdontopedClinIntegr, João Pessoa, 2011; 11(4):593-99

9. Rosário SSD, Fernandes APNL, Batista FWB, Monteiro AI. Acessibilidade de crianças com deficiência aos serviços de saúde na atenção primária Rev. Eletr. Enf. 2013; 15(3):740-6 Disponível em: http://dx.doi.org/10.5216/ree.v15i3.19272.doi:10.5216/ree.v15i3.19272

10. Nasiloski SCN, et al. Avaliação das condições periodontais e de higiene bucal em escolares com transtornos neuropsicomotores Rev. Odontol. UNESP, 2015; 44(2):103-107

11. Amaral FLJS, et al. Acessibilidade de pessoas com deficiência ou restrição permanente de mobilidade ao SUS. Ciência \& Saúde Coletiva, 2012; 17(7):1833-1840

12. Castro, SS et al. Acessibilidade aos serviços de saúde por pessoas com deficiência. Revista de Saúde Publica, 2011; 45(1):99-105

13. Silveira ER, et al. Educação em Saúde Bucal Direcionada aos Deficientes Visuais. Rev. Bras. Ed. Esp., Marília, 2015; 21(2):289-298

14. Antunes MH, Rocha EF. Desbravando novos territórios: incorporação da Terapia Ocupacional na estratégia da Saúde da Família no município de São Paulo e a sua atuação na atenção à saúde da pessoa com deficiência - no período de 2000-2006. Revista Terapia Ocupacional, 2011; 22(3):270-278

15. Bernardes LCG, Araújo TCCF. Deficiência, políticas públicas e bioética: percepção de gestores públicos e conselheiros de direitos. Ciência \& Saúde Coletiva, 2012; 17(9):24352446

16. Araújo DB, Menezes LMB, Sousa DL. Atenção secundária em saúde bucal e a implementação dos Centros de Especialidades Odontológicas em um estado do Nordeste, Brasil. RGO, 2012; 60(1):49-54

17. Oliveira YCA, Coura AS, Costa GMC et al. Comunicação entre profissionais de saúdepessoas surdas: revisão integrativa .Rev. enferm UFPE online. Recife, 2015; 9(2):957-64

18. Mendes ACG, Miranda GMD, Figueiredo KEG, Duarte PO, Furtado BMASM. Acessibilidade aos serviços básicos de saúde: um caminho ainda a percorrer. Ciências saúde coletiva [online]. 2012; 16(17):11:2903-12

Availablefrom:http://www.scielo.br/scielo.php?script=sci_arttext\&pid=S14138123201200110 0007\&lng=en\&nrm=iso 
19. Maia ER, Pagliuca LM, Almeida PC. Aprendizagem do agente comunitário de saúde para identificar e cadastrar pessoas com deficiência. Acta Paulista de Enfermagem, 2014; 27(4):326-32

20. Aragão AKR et al. Acessibilidade da Criança e do Adolescente com Deficiência na Atenção Básica de Saúde Bucal no Serviço Público: Estudo Piloto. Pesquisa Brasileira Odontopediatria Clinica Integrada, 2011; 11(2):159-164

21. Fiorati RC, Elui VMC. Determinantes sociais da saúde, iniquidades e inclusão social entre pessoas com deficiência. Revista Latino-Americana de Enfermagem, 2015; 23(2):329-36

22. BRASIL. Ministério da Saúde (MS). Secretaria de Atenção à Saúde. Departamento de Atenção básica. Coordenação Nacional de Saúde bucal. Diretrizes da Política Nacional de Saúde Bucal. Brasília: MS; 2004.

23. Marta SN. Programa de assistência odontológica ao paciente especial: uma experiência de 13 anos. RGO - Revista Gaúcha Odontologia. Porto Alegre, 2011; 59(3):379-385

24. Machado FCA et al. Fatores relacionados ao desempenho de Centros de Especialidades Odontológicas. Ciência \& Saúde Coletiva, 2015; 20(4):1149-1163

25. Menchaca MHR, Alanis TMG, Silva RG. Guia para el cuidado de La salud oral en pacientes com necesidad de cuidados especiales de saluden México. Rev. ADM, 2011; 68(5):222-8

26. Nacamura CA et al. Síndrome de Down: inclusão no atendimento odontológico municipal. Faculdade de Odontologia de Lins/Unimep, 2015; 25(1):27-35

27. Gardens SJ, Krishna M, Vellappally S, Alzoman H, Halawany HS, Abraham NB et al. Oral health survey of 6-12-year-old children with disabilities attending special schools in Chennai, India. Int J PaediatrDent, 2014; 24(6):424-33

28. Castilho LS et al. O trabalho voluntário e a educação do cirurgião-dentista: a experiência de um projeto de extensão odontológico. Em extensão, Uberlândia, 2014; 13(2):162-170

29. Lawrence $\mathrm{H}$ et al. Acesso à Saúde Bucal Pública pelo Paciente Especial: A Ótica do Cirurgião-Dentista. Revista Brasileira de Promoção da Saúde, 2014; 27(2):190-197

30. Castro SS, Cieza A, Cesar CL. Problems with accessibility to health services by persons with disabilities in São Paulo, Brazil. DisabilRehabil., 2011; 33(17-18):1693-8 